\title{
VIBRATION OF QUARTZ CRYSTAL PLATES
}

\author{
BY \\ R. P. JERRARD* \\ University of Illinois
}

1. Introduction. Among the most difficult vibration problems are those arising in the analysis of quartz plate vibration. In the first place the quartz crystal is highly anisotropic. Secondly, the most pressing problems involve plates vibrating in complicated modes where simple plate theory does not apply. Finally, many of these plates are of non-uniform thickness, being bevelled at the edges or ground into convex form. At present, the best guides to an understanding are the work of Mindlin and Newman [1], [2], Sykes [3], and Ekstein [4].

In particular, Mindlin has developed a theory of elasticity for plates based on expansion of the displacement functions in powers of the distance from the center plane of the plate. He has successfully used this theory in describing the interaction of the fundamental thickness shear mode with high harmonics of the lengthwise flexure modes.

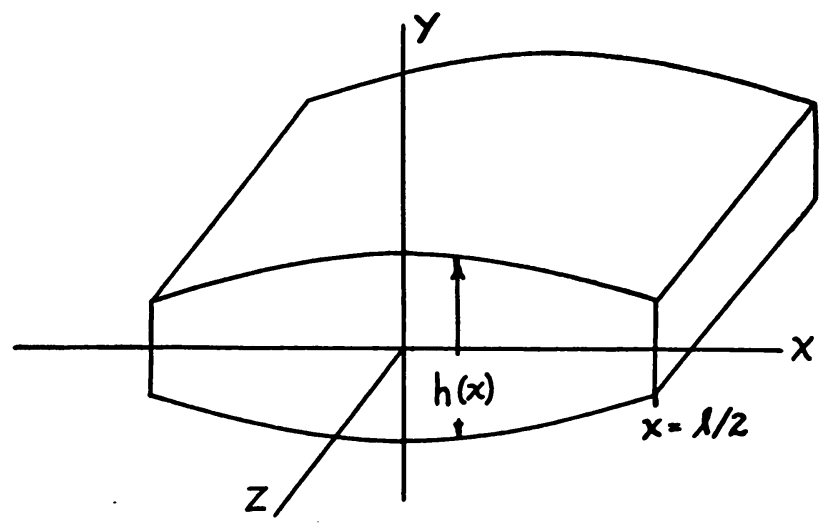

Fig. 1. Coordinate axes and shape of crystal plate.

Referring to Fig. 1, the crystal is said to vibrate in fundamental thickness shear when the top and bottom surfaces are moving in opposition to one another, and their motion is predominantly in the $x$-direction. A lengthwise flexure mode is one in which the middle surface of the crystal has a $y$-displacement like $\sin m x$. Interaction between the two modes takes place only when their frequencies are about equal, and since the crystal is quite thin, this will occur only for high harmonics of the flexure mode.

Very little analysis of plates with non-uniform thickness has been made, though many such plates are manufactured. It is found empirically that with a plate of convex shape the desired thickness shear modes are purer, that is, freer of distortion which is apparently due to coupling with other modes, particularly the high order lengthwise

*Received April 17, 1959; revised manuscript received September 11, 1959. This work was carried out while the author was a staff member at Bell Telephone Laboratories, Whippany, N. J. 
flexure modes. It is also found that with a convex, or "contoured" plate the surface displacement near the edges is very small compared to the displacement at the center.

In this paper, an analysis of such contoured plates is made. It is found that with one further approximation, Mindlin's plate theory can be applied to a certain class of contoured plates. These solutions have been obtained, and the frequencies computed for certain plates. The analysis is given in Sec. 2, and the numerical results in Sec. 3 .

2. Analysis of contoured plate vibration. We take as a starting point the plate theory developed by Mindlin [2]. The coordinate system is shown in Fig. 1. The I. R. E. standard notation is used for the stresses, displacements, and the elastic constants of quartz.

There are two essential features of the solution. First, it is assumed that $T_{2}$, the stress in the $y$-direction, is zero. This is reasonable for thin plates. Second, the form of the displacement components is chosen to be

$$
\begin{aligned}
u & =y \psi(x, z, t), \\
v & =\eta(x, z, t), \\
w & =y \varphi(x, z, t),
\end{aligned}
$$

where $\psi, \eta$, and $\varphi$ are functions to be determined. In the expansion of the displacements in powers of $y$ we use at most only the linear term. We later specialize to the case where $w$ vanishes. It is seen that the $y$ dependence of the displacements has been prescribed so as to permit a thickness-shear type of motion.

The general procedure for obtaining equations of motion is as follows. There are six elastic stress-strain relations, one of which is altered when $T_{2}$ is set equal to zero. This equation is solved for $\partial v / \partial y$, and this quantity is eliminated from the remaining five equations. The assumed displacement functions of Eq. (1) are substituted into the five equations, which are then integrated with respect to $y$. This integration introduces three stress couples and two stress resultants which are defined as follows:

$$
\begin{gathered}
\left(M_{x}, M_{z}, M_{x z}\right)=\int_{-h / 2}^{h / 2}\left(T_{1}, T_{3}, T_{5}\right) y d y, \\
\left(Q_{x}, Q_{z}\right)=\int_{-h / 2}^{h / 2}\left(T_{6}, T_{4}\right) d y .
\end{gathered}
$$

The five stress-strain relations now express these five plate stress components in terms of the functions $\psi, \eta, \varphi$ of Eq. (1) as follows:

$$
\begin{aligned}
M_{x} & =D_{1} \frac{\partial \psi}{\partial x}+D_{2} \frac{\partial \varphi}{\partial z}, \\
M_{z} & =D_{2} \frac{\partial \psi}{\partial x}+D_{3} \frac{\partial \varphi}{\partial z}, \\
M_{x z} & =D_{5}\left[\frac{\partial \varphi}{\partial x}+\frac{\partial \psi}{\partial z}\right], \\
Q_{z} & =D_{4}\left[\varphi+\frac{\partial \eta}{\partial z}\right], \\
Q_{x} & =D_{6}\left[\psi+\frac{\partial \eta}{\partial x}\right],
\end{aligned}
$$


where

$$
\begin{aligned}
& D_{1}=\left[C_{11}-C_{12}^{2} / C_{22}\right] h^{3} / 12, \\
& D_{2}=\left[C_{13}-C_{12} C_{23} / C_{22}\right] h^{3} / 12, \\
& D_{3}=\left[C_{33}-C_{23}^{2} / C_{22}\right] h^{3} / 12, \\
& D_{4}=\left[C_{44}-C_{24}^{2} / C_{22}\right] h \pi^{2} / 12, \\
& D_{5}=C_{55} h^{3} / 12, \\
& D_{6}=C_{66} h \pi^{2} / 12 .
\end{aligned}
$$

The multiplier $\pi^{2} / 12$ in $D_{4}$ and $D_{6}$ was introduced by Mindlin as a correction factor. The constants $C_{i j}$ are the elastic constants of quartz.

We must also make use of the three stress equations of motion. By integrating them with respect to $y$, one can write these equations in terms of the five plate stress components given above. In carrying out this integration, and in the previous integration, it is necessary to apply the boundary conditions $T_{4}=T_{6}=0$ on $y= \pm h / 2$. These conditions simply state that the shear stresses vanish on the top and bottom of the plate. Actually, $T_{4}$ and $T_{6}$ are the shear stresses on a surface normal to the $y$-axis, and the contoured surfaces are not normal to the $y$-axis. The error involved is small if the contouring is limited.

The equations of motion in terms of plate stress components can be written fairly simply when a factor $e^{i \omega t}$ is introduced. They are

$$
\begin{aligned}
& \frac{\partial M_{x}}{\partial x}-Q_{x}+\frac{\partial M_{x z}}{\partial z}+\rho h^{3} \omega^{2} \psi / 12=0 \\
& \frac{\partial Q_{x}}{\partial x}+\frac{\partial Q_{z}}{\partial z}+\rho h \omega^{2} \eta=0 \\
& \frac{\partial M_{z}}{\partial z}-Q_{z}+\frac{\partial M_{x z}}{\partial x}+\rho h^{3} \omega^{2} \varphi / 12=0 .
\end{aligned}
$$

We now assume that the motion of the plate is independent of $z$. This would be the situation if, for instance, the plate were infinitely long in the $z$ direction. With this assumption, we find that $\varphi, M_{x z}$, and $Q_{z}$ all vanish, so the third of Eqs. (5) is automatically satisfied. The only remaining independent variable is $x$, and we denote differentiations with respect to $x$ by primes. Equations (5) reduce to the following two equations

$$
\begin{aligned}
& \left(D_{1} \psi^{\prime}\right)^{\prime}-D_{6}\left(\psi+\eta^{\prime}\right)+\rho h^{3} \omega^{2} \psi / 12=0, \\
& {\left[D_{6}\left(\psi+\eta^{\prime}\right)\right]^{\prime}+\rho h \omega^{2} \eta=0 .}
\end{aligned}
$$

These are the equations that must be solved, subject to certain boundary conditions at the edges of the plate, $x= \pm l / 2$.

At this point Mindlin and Forray [5] obtained an approximate solution by putting $D_{1}=0$, and introducing a correction factor for $D_{6}$. Their solution approximates the frequencies of the "uncoupled" shear and flexure modes. In the present paper, another 
solution is obtained which retains the coupling between modes. This solution requires that the approximation

$$
h^{3} \cong h \bar{h}^{2},
$$

where $\bar{h}$ is a constant "effective" thickness, be used wherever $h^{3}$ appears in Eqs. (6). The effect of this is to alter the stiffness, bringing it closer to the stiffness of a flat plate. In other words, this approximation will reduce the calculated effect of contouring.

We proceed to carry out the differentiations indicated in Eqs. (6), first introducing the notation

$$
\begin{aligned}
D_{10} \frac{h^{3}}{12} & =D_{1}, \\
D_{60} h & =D_{6},
\end{aligned}
$$

so that $D_{10}$ and $D_{60}$ do not depend upon the thickness. The equations of motion (6) can now be written

$$
\begin{aligned}
& \psi^{\prime \prime}+\frac{\left(h^{3}\right)^{\prime}}{h^{3}} \psi^{\prime}-\frac{12 D_{60}}{D_{10}} \frac{\psi+\eta^{\prime}}{h^{2}}+\frac{\rho \omega^{2} \psi}{D_{10}}=0, \\
& \psi^{\prime}+\eta^{\prime \prime}+\frac{h^{\prime}}{h}\left(\psi+\eta^{\prime}\right)+\frac{\rho \omega^{2} \eta}{D_{60}}=0 .
\end{aligned}
$$

This pair of equations can be reduced to a single equation by first introducing the approximation (7), and second assuming a solution of (9) of the form

$$
\psi=(\sigma-1) \eta^{\prime}
$$

where $\sigma$ is a constant.

After using (7) and eliminating $\psi$ by means of (10), Eqs. (9) become

$$
\begin{aligned}
& \eta^{\prime \prime \prime}+\frac{h^{\prime}}{h} \eta^{\prime \prime}+\left[\frac{\rho \omega^{2}}{D_{10}}-\frac{\sigma}{\sigma-1} \frac{12 D_{60}}{D_{10} \bar{h}^{2}}\right] \eta^{\prime}=0, \\
& \eta^{\prime \prime \prime}+\frac{h^{\prime}}{h} \eta^{\prime \prime}+\left[\frac{\rho \omega^{2}}{\sigma D_{60}}+\left(\frac{h^{\prime}}{h}\right)^{\prime}\right] \eta^{\prime}=0,
\end{aligned}
$$

where the second of Eqs. (9) has been differentiated.

These two equations can now be made identical by adjusting the constant $\sigma$. The value of $\sigma$ which makes them identical is obtained by equating the third terms of the two equations to each other

$$
\frac{\rho \omega^{2}}{D_{10}}-\frac{\sigma}{\sigma-1} \frac{12 D_{60}}{D_{10} h^{2}}=\frac{\rho \omega^{2}}{\sigma D_{60}}+\left(\frac{h^{\prime}}{h}\right)^{\prime} .
$$

We note that if $\sigma$ is to be a constant, then the right hand term in (12) must be constant. This restricts the form of $h$, for then we must have

$$
\left(\frac{h^{\prime}}{h}\right)^{\prime}=\text { constant. }
$$

This is a differential equation in $h(x)$, which can easily be solved to yield

$$
h(x)=h_{0} \exp \left(k_{1} \frac{x^{2}}{2}+k_{2} x\right) .
$$


When $h(x)$ is any expression of the form (14), the approximate solution can be carried through with no further assumptions. We will consider only the family of crystal shapes that result when $k_{2}=0, k_{1}<0$.

Returning to the constant $\sigma$, one can solve the quadratic equation (12) for $\sigma$ to obtain

$$
\frac{1}{\sigma}=\frac{1}{2}\left\{1+g-\lambda\left(\frac{h^{\prime}}{h}\right)^{\prime} \pm\left(\left[1-g+\lambda\left(\frac{h^{\prime}}{h}\right)^{\prime}\right]^{2}+\frac{4 \lambda g}{R}\right)^{1 / 2}\right\},
$$

where

$$
\begin{aligned}
g & =\frac{D_{60}}{D_{10}}, \\
\lambda & =\frac{D_{60}}{\rho \omega^{2}}, \\
R & =\frac{\bar{h}^{2}}{12} .
\end{aligned}
$$

Note that the frequency occurs in the constants $\lambda, \sigma$.

We now have one solution of Eqs. (9), which is given by Eqs. (10) and (15). The remaining equation can be taken as the second of (9), which is now written in the form

$$
\eta^{\prime \prime}+\frac{h^{\prime}}{h} \eta^{\prime}+\frac{1}{\lambda \sigma} \eta=0 .
$$

There are two values of $1 / \sigma$ given by Eq. (15), and since (17) is of the second order, there will be two solutions of (17) for each value of $\sigma$. The exact form of (17) depends upon the form chosen for the thickness $h(x)$.

The boundary conditions are given by Mindlin [2]; the edges $x= \pm l / 2$ are free, and the boundary conditions are

$$
M_{x}=Q_{x}=0, \quad x= \pm l / 2 .
$$

Solutions of (17) must satisfy these conditions. Using Eq. (3), the conditions can be rewritten in the form

$$
\left.\begin{array}{r}
\psi^{\prime}=0 \\
\psi+\eta^{\prime}=0
\end{array}\right\} \text { at } x= \pm l / 2 .
$$

Thus, corresponding to the four solutions of Eq. (17) (two for each $\sigma$ ), there are four boundary conditions to determine the arbitrary constants.

We can now indicate the complete solution to the problem. Taking $h(x)$ as given by Eq. (14), the differential equation (17) becomes

$$
\eta^{\prime \prime}+\left(k_{1} x+k_{2}\right) \eta^{\prime}+\frac{1}{\lambda \sigma} \eta=0 .
$$

For each $\sigma$ this equation will have two solutions, one even in $x$, the other odd in $x$. Since the fundamental thickness shear mode of vibration will couple only with the even flexure modes, we consider only the solutions to (17) which correspond to even flexural modes. For these modes, $\eta$ is an odd function of $x$, so our solutions will be of the form

$$
\eta=A_{1} f\left(\sigma_{1}, x\right)+A_{2} f\left(\sigma_{2}, x\right),
$$


where $f(\sigma, x)$ is the odd solution to Eq. (20). We need apply the boundary conditions only at $x=l / 2$, for since the solution is odd in $x$, the conditions will automatically be satisfied at the other edge.

From (21) and (10), the form of $\psi$ will be

$$
\psi=A_{1}\left(\sigma_{1}-1\right) f^{\prime}\left(\sigma_{1}, x\right)+A_{2}\left(\sigma_{2}-1\right) f^{\prime}\left(\sigma_{2}, x\right) .
$$

Substituting these expressions for $\psi$ and $\eta$ into the boundary conditions (19), one gets two linear, homogeneous algebraic equations in the two unknown constants $A_{1}$ and $A_{2}$. To obtain an equation for the frequency, one sets the determinant of the coefficients in the two equations to zero. This latter condition, which provides the frequency equation, must be satisfied if the boundary condition equations are to have a solution. Proceeding in this way, one gets the frequency equation

$$
\sigma_{1}\left(\sigma_{2}-1\right) f^{\prime \prime}\left(\sigma_{2}, l / 2\right) f^{\prime}\left(\sigma_{1}, l / 2\right)-\sigma_{2}\left(\sigma_{1}-1\right) f^{\prime \prime}\left(\sigma_{1}, l / 2\right) f^{\prime}\left(\sigma_{2}, l / 2\right)=0 .
$$

The only unknown in this equation is the frequency $\omega$, which appears in both $\sigma_{1}$ and $\sigma_{2}$. In general, Eq. (23) is a transcendental equation, and will have an infinite number of roots. This is to be expected, since any crystal of fixed dimensions can theoretically vibrate in an infinite number of overtone modes. Thus for any given set of dimensions, one can solve Eq. (23) to obtain the natural frequencies of vibration.

3. Numerical results-conclusions. In order to obtain the frequency spectrum for this class of quartz plates, we must discover the distribution of roots of the transcendental equation (23). This equation involves the function $f(\sigma, x)$ which is the odd solution of the differential equation (20). The solutions of this equation are certain confluent hypergeometric functions [6], which are not available in tabular form. Thus the task of finding the roots of (23) involves a very lengthy calculation.

The expression on the left side of (23) depends, aside from elastic constants, upon a total of three dimensionless parameters; when two of these are specified, one can obtain a sequence of values of the third parameter at which (23) has a root. This must be done for a large number of combinations of the first two parameters in order to obtain a good picture of the frequency spectrum. An IBM 704 computer was used to carry out this calculation. The required functions were expressed as series, and a procedure similar to Newton's method was used to find the roots.

The left side of (23) can be expressed in terms of the four parameters

$$
\omega / \bar{\omega}, \quad l / \bar{h}, \quad k_{1} l^{2} / 8, \quad g .
$$

Here $l$ is the length of the crystal plate in the $x$-direction, $g$ (defined in Eq. (16)) is equal to 0.283 for $A T$-cut quartz, $\bar{h}$ is an average thickness as defined below, and $\omega$ is the radian frequency of vibration. Also $\bar{\omega}$ is the frequency in fundamental thickness shear vibration of an infinite flat plate of thickness $\bar{h}$. We see from Eq. (14) that

$$
k_{1} l^{2} / 8=\log \left[h(l / 2) / h_{0}\right]=\log r,
$$

where $r$ is the ratio of edge thickness to center thickness.

The effective thickness $\bar{h}$ will be defined by

$$
\bar{h}=\left[\frac{2}{l} \int_{0}^{l / 2} h^{2}(x) d x\right]^{1 / 2} .
$$


This definition is obtained by minimizing the integrated squared difference between $h^{2}(x)$ and $\bar{h}^{2}$, since we are approximating the former by the latter. For $h(x)$ as given by Eq. (14) we find $\left(k_{1}<0\right)$

$$
\bar{h}=h_{0}\left(-\pi / 2 k_{1} l^{2}\right)^{1 / 4}\left[\operatorname{erf}\left(-k_{1} l^{2} / 2\right)^{1 / 2}\right]^{1 / 2} .
$$

Experimental data would show whether definition (26) or some other weighted average would be better. However, the definition of $\bar{h}$ is not used in the computations described below.

The calculation of frequencies was carried out for five values of $r$, namely 1.0, 0.778, $0.606,0.448$, and 0.368 . That is, for each of the values of $r$, the frequency ratio $\omega / \bar{\omega}$ was obtained as a function of $l / \bar{h}$. Figure 2 shows the resulting frequency spectrum when $l / \bar{h}$ is in the neighborhood of twenty-eight.

When $\log r$ is zero $(r=1)$, the curve coincides with Mindlin's result [2] for the flat plate. The curves have the characteristic structure that also appears in the flat plate solution. The more nearly horizontal portions correspond to vibration which is predominantly thickness shear, while the more vertical portions correspond to flexural vibration. This analysis does not show any marked change in the interaction between these modes as the contouring is increased; the main effect is to bring the frequency ratio closer to unity.

We may assume that if the flexure modes were completely suppressed, the resulting frequency spectrum could be approximated by drawing nearly horizontal curves through the inflection points of the curves of Fig. 2. This approximation to the frequency ratio

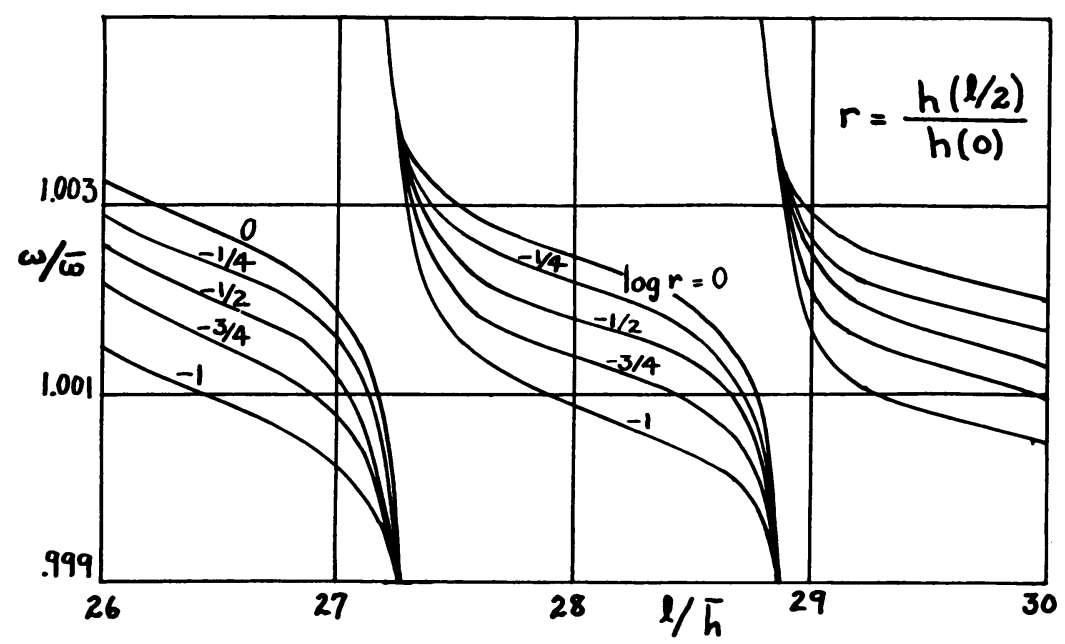

Fig. 2. Frequency ratio as a function of length-to-thickness ratio for different degrees of contouring of AT-cut crystal.

$\omega / \bar{\omega}$ (sometimes called the "frequency constant") is often made, in order to show the gross behavior of the thickness shear frequency as a function of $l / \bar{h}$. A set of such curves for the five values of $r$ is shown on Fig. 3. This shows much more clearly how the computed effect of increased contouring is to bring the frequency ratio close to unity.

The effect of contouring on the first even overtone mode is shown in Fig. 4. This is the first overtone mode for which $u$ is an even function of $x$. Referring to Fig. 1, the 


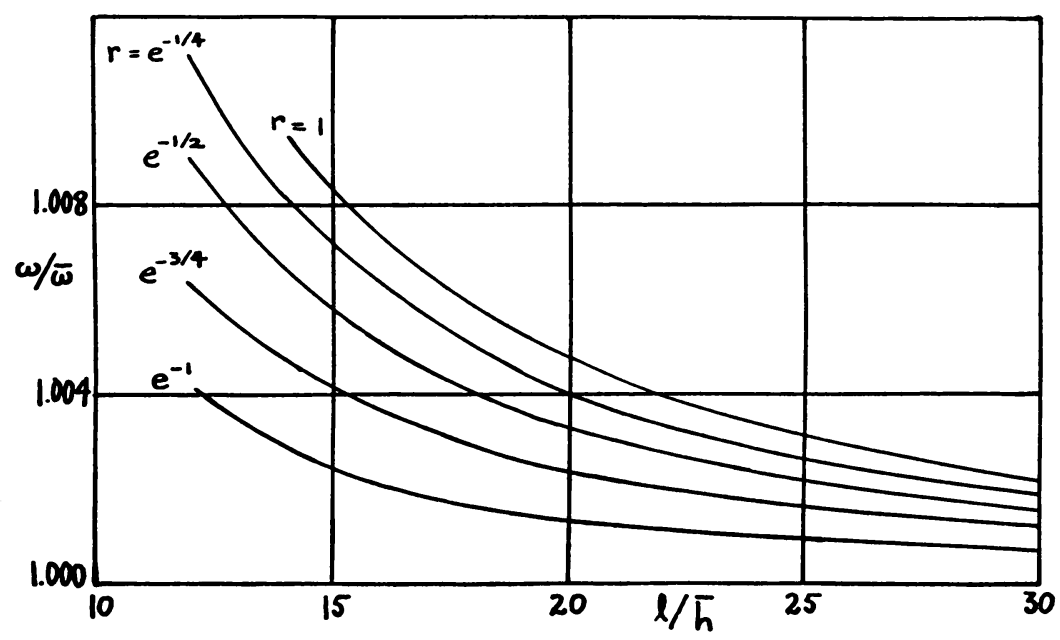

FIG. 3. Approximate frequency ratio for different degrees of contouring of AT-cut crystal.

motion of the surfaces is still parallel to the $x z$-plane, and the displacement $u$ is still proportional to $y$. However, in this mode the center of the top surface moves in opposition to its edges; the displacement $u$ is a function like $y \cos (2 \pi x / l)$. In Fig. 4 the flattened portions of the curve with a frequency ratio near 1.018 correspond to this mode. It is seen that for this overtone mode the computed effect of contouring is less than for the fundamental. Thus the results show that the separation in frequency between the fundamental and the first even overtone mode is slightly larger for a contoured quartz plate.

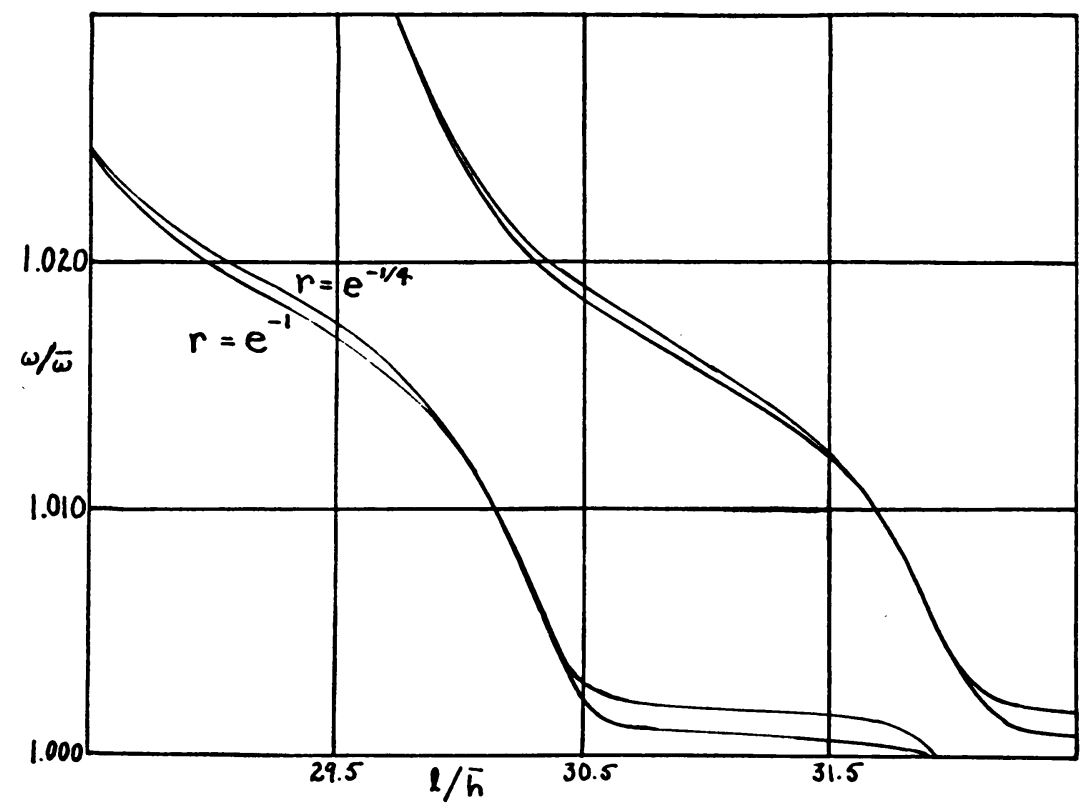

Fig. 4. Frequency ratio for two degrees of contouring showing the first even overtone mode. 
Some general remarks may be made about this solution. First, it is in agreement with experiment in that the calculated displacement $u$ on the surface does decrease as one proceeds from the center towards the edges. Though at any constant height $y$ the displacement is greater near the edge, the decreasing height of the surface as one moves away from the center is more than enough to offset this. Second, the approximation (7) makes possible the simple solution (10) of the differential equations. The relation (10) essentially says that the fundamental thickness shear mode, for which $u$ is an even function of $x$, couples only with alternate flexural modes for which $v$ is an odd function of $x$. This simple relationship does not hold when the approximation (7) is not made. Nonetheless, experimental evidence seems to show only this coupling with alternate flexural modes.

\section{BIBLIOGRAPHY}

1. E. G. Newman and R. D. Mindlin, Vibrations of a monoclinic crystal plate, J. Accoust. Soc. Amer. 29, 11, 1206 (1957)

2. R. D. Mindlin, Thickness-shear and flexural vibrations of crystal plates, J. Appl. Phys. 22, 3, 316 (1951)

3. Quartz crystals in electrical circuits, edited by R. A. Heising, Van Nostrand, 1946, pp. 205-248

4. H. Ekstein, High frequency vibrations of thin crystal plates, Phys. Rev. 68, 1, 11 (1945)

5. R. D. Mindlin and M. Forray, Thickness-shear and flexural vibrations of contoured crystal plates, J. Appl. Phys. 25, 1, 12 (1954)

6. Jeffreys and Jeffreys, Methods of mathematical physics, 3rd ed., Cambridge University Press, 1956, p. 620 